\title{
Allometric scaling models: history, use, and misuse in translating resveratrol from basic science to human clinical applications
}

\author{
James M. Smoliga ${ }^{1}$ and Otis L. Blanchard ${ }^{2}$ \\ ${ }^{1}$ Department of Physical Therapy, High Point University, High Point, NC 27268, USA; ${ }^{2}$ Ace \\ Laboratories, Seattle, WA 98059, USA \\ Corresponding Author: James M. Smoliga, DVM, PhD. Associate Professor of Physiology, \\ Department of Physical Therapy. High Point University, High Point, NC 27407, USA \\ Submission Date: March $9^{\text {th }}$, 2017, Acceptance Date: May $29^{\text {th }}, 2017$, Publication Date: May \\ $30^{\text {th }}, 2017$ \\ Citation: Smoliga J.M., Blanchard O.L. Allometric scaling models: history, use, and misuse in \\ translating resveratrol from basic science to human clinical applications. Functional Foods in \\ Health and Disease 2017; 7(5); 338-352.
}

\begin{abstract}
Determination of the first-in-human and pharmacologically active dosage for drugs and nutraceutical compounds is a critical step in study design and product development. Allometric scaling is a form of mathematic modeling commonly used to convert dosages between species. While allometric scaling allows for quick and straightforward conversions between species, it is often misunderstood and misused in translational clinical applications. This is readily demonstrated in the case of resveratrol - a polyphenol which is found in red wine. In the past decade, a considerable amount of research has emerged regarding the health benefits of the resveratrol supplementation. Although data from rodent models suggests that resveratrol can have major effects on cardiometabolic and neurologic health, human clinical trials have had mixed results. While some human clinical trials have yielded encouraging results, a few noteworthy trials have reported that seemingly appropriate allometry-derived dosages of resveratrol did not provide the expected health benefits reported in animal models. In this article, we discuss the history of various models within allometry, including their advantages, disadvantages, and nuances from a clinical perspective. This historical information will provide some insight into why dosages recommended from allometric scaling are appropriate in some circumstances and inappropriate in others. We will then demonstrate how allometric models have been utilized to translate dosages of resveratrol from rodent models into the dosages recommended for human clinical trials. Pharmacokinetic data from various human clinical trials were summarized and compared to data predicted from allometric models. Data from selected human clinical trials were then synthesized to demonstrate the dosage-dependent effects of resveratrol and to provide further insight into the appropriate use of allometric models for
\end{abstract}


selecting resveratrol dosage. Altogether, this information will promote a greater understanding of the role of allometric scaling in dose selection and provide an explanation for some of the apparent inconsistencies in translational research regarding resveratrol.

Keywords: allometric scaling, dose conversion, bioavailability, pharmacokinetics, resveratrol

\section{INTRODUCTION}

Appropriate interspecies dosage translation is a key component in moving nutraceutical and pharmaceutical research from basic science laboratories into clinical settings. It is wellestablished that laboratory animal experiments are valuable in determining the dosage at which physiological benefits and adverse events occur, in addition to the magnitude of benefits and risks. While laboratory animals provide insight into the absorption, metabolism, tissue distribution, and excretion of novel nutritional and therapeutic products, they do not provide direct or exact information regarding the safe and effective dosages to be used in the initial stages of human clinical trials. Accordingly, various physiologically-based pharmacokinetic (PBPK) modeling methods are utilized to predict outcomes [3, 4]. These can be utilized from in vitro data to predict drug candidate metabolism and distribution. While advanced methods, such as PBPK, require more data from a given molecule and species to achieve more accurate predictions, the time and effort involved in this process may be too complex and resourceintensive for the majority of small studies conducted in small labs. As such, the proper use of allometry may allow for a straightforward dosage translation at a low cost. One of the oldest, and perhaps most commonly misunderstood, methods to estimate dosages between species is allometric scaling.

Allometric scaling is a method based on correlational relationships between species of different sizes which share common physiological characteristics [5]. The allometric formula originates from work in metabolic scaling, where an equation is developed to relate the metabolic rate and body sizes of various species on a double logarithmic scale [6-8]. This concept originated in the 1800's and was based on the concept that body surface area (BSA) influences environmental heat transfer. Consequently, it was hypothesized that mammals must produce heat at a rate equivalent to the rate of surface heat loss. According to this crude measure, surface area was found to be related to body mass to the two-thirds power (Equation 1) [5]:

\section{Equation 1: Surface area $=$ constant $x$ Mass $^{2 / 3}$}

The hypothesis of a ratio of metabolism to surface area within the animal kingdom was very popular in the late 1800 's Western scientific community, where every theory conflicting with the surface law was assumed to be wrong [5,9]. In the Victorian-era scientific and cultural discussion, reports in which the measured surface area of a species did not match the reported metabolic rate were simply dismissed as "error" [9]. As much as allometric scaling is used for relating body mass or size to metabolism, allometry has been used in scaling other physiologic parameters across species and has been expanded to estimate pharmacokinetic parameters. The allometric approach to drug dosages requires pharmacokinetic data to be available for at least two model species (or preferably three) [7, 10]. In the allometric formula (Equation 2), $y$ is the pharmacokinetic parameter of interest and $W$ represents body weight. The parameter of interest is 
explored in three species of differing body weight, with the data being plotted on a log-log scale with body mass on the $x$-axis and the parameter of interest on the $y$-axis [6-8, 10]. From the scale, the y-intercept is the coefficient $(a)$ in the allometric equation and the exponent $(b)$ is the slope $[6-8,10]$. The allometric equation can then be computed. The allometric equation can be solved using the body mass of a human, allowing for prediction of the pharmacokinetic parameter of interest.

\section{Equation 2: $\quad y=a W^{b}$}

The exponent of the allometric equation can provide information regarding which allometric scaling model should be used in prediction (e.g., simple allometry vs. other allometric methods). Various modifications and corrective factors for prediction can be used to quickly approximate the range of human pharmacokinetic parameters, including circulation time, clearance, or blood flow $[7,8]$. For instance, drug clearance may be best estimated by simple allometry when the exponent ranges from 0.55 to 0.70 , but if it is greater than this other allometric models can be used. These include scaling to maximal lifespan potential or scaling to brain weight. A detailed discussion of this "rule of exponents," and how it was derived is available elsewhere [5, 11] demonstrates the nuances of choosing a scaling method. Furthermore, it cannot be emphasized enough that these methods are meant to only provide an estimate of pharmacokinetic parameters, with substantial error (30-50\%) thereby being considered acceptable [5]. Despite the high error, the cost of human trials allows the low-cost method to be practical for general prediction from multiple animal studies [7, 8, 10]. There are also published methods to predict clearance, volume of distribution, and excretion by various modifications to allometry, with various degrees of success $[8,10,12]$. The exponents and accuracy will vary on the number of species used in the equation, physical properties of the drug, and interspecies differences in physiology [13]. For example, the use of allometry in practice can be found in the pharmacokinetic investigation of MI-219[14]. Allometry is purely correlative, is not based on physiology, and does not take into consideration interspecies differences contributing to the high error rate and multiple other pitfalls $[8,10]$.

As previously stated, the ability to use pharmacokinetic data from animal species to predict how a drug or nutraceutical product will behave in humans is valuable in the developmental stages. Thus, allometric scaling is indeed useful for predicting the pharmacokinetic properties of drugs and nutrients in humans when data pharmacologic data are not available.

\section{ALLOMETRIC SCALING OF A NUTRACEUTICAL PRODUCT - RESVERATROL AS AN EXAMPLE}

Numerous bioactive compounds are isolated from plant species and are tested in cell culture for their potential biomedical usage, eventually leading to investigations in laboratory animal models. When promising results are consistently identified in model species, the need to determine an appropriate dose for humans arises. One of the relatively recent high-profile cases of attempted allometric scaling for interspecies dosage translation is that of resveratrol.

\section{Brief History of Resveratrol as a Nutraceutical Intervention}


Resveratrol is a polyphenol found in various nuts and berries, being most well-known as the "red wine antioxidant." Resveratrol was first described in 1939 [15] and began to receive some attention in the botanical literature in the mid-1970's [16]. However, the health effects of resveratrol received little consideration until 1992, when it was suspected to be one of the active chemicals responsible for the observed cardioprotective effects of red wine consumption [17]. Resveratrol began to receive major attention in 1997, after it was discovered to have anti-cancer activity [18]. Within the next decade, resveratrol continued to receive notoriety after a series of Harvard-based studies demonstrated that it was the most potent activator of a gene (SIRT1) associated with anti-aging benefits of caloric restriction [19, 20]. After a 2006 paper revealed resveratrol improved health and survival in obese mice [21], resveratrol became a major molecule of interest for the pharmaceutical and dietary supplement industry. Unfortunately, confusion over the ideal dosage for humans may have caused a number of setbacks in the development of clinical applications for resveratrol.

\section{Conflicting Opinions on Resveratrol Dosage for Humans}

In their groundbreaking 2006 paper, Baur et al reported that mice were fed two different concentrations of resveratrol, $5.2 \pm 0.1 \mathrm{mg} / \mathrm{kg}$ and $22.4 \pm 0.4 \mathrm{mg} / \mathrm{kg}$ per day, noting that the higher dosage was more effective than the lower dosage in eliciting a number of beneficial health effects. Thus, the authors focused their analysis on the more efficacious (higher) dose. Additionally, they stated that these daily doses were realistically attainable in humans. Using the $22.4 \mathrm{mg} / \mathrm{kg}$ value, this could theoretically equate to about $1500 \mathrm{mg}$ of resveratrol per day for a $70 \mathrm{~kg}$ human. However, in 2008, Reagan-Shaw et al, published a paper which argued that Baur et al were incorrect in using this one-to-one dosage translation, and that "allometric dose translation" was necessary [22]. As correctly stated by Reagan-Shaw et al, it is incorrect to assume that a direct $\mathrm{mg} / \mathrm{kg}$ oral dosage from chow directly correlates to an oral dosage of similar effect in humans [22-24]. Mice cannot simply be thought of as small humans in their use as a model species $[25,26]$. There are physiological differences which may allow for a much higher clearance rate of small molecules from mice compared to humans [7] and the literature is filled with examples where allometric conversions do not hold true [5]. As such, the authors argued for the use of BSA scaling, and using the formulae provided by the FDA, report that Baur's data actually translates to do a daily dosage of $1.82 \mathrm{mg} / \mathrm{kg}$, or $\sim 130 \mathrm{mg}$ per day for a $70 \mathrm{~kg}$ human. In other words, the dosage recommended by Baur et al is about twelve times greater than that recommended by Reagan-Shaw et al. The discrepancies in recommended dosage between these sources simply promoted confusion in the clinical trials which followed.

Given the discrepancies in interspecies dosage conversion, it is not surprising that human clinical trials exploring resveratrol interventions have used a wide variety of dosages (Figure 1), with most studies using dosages closer to Reagan-Shaw et al's 2008 recommendation, rather than Baur's 2006 recommendation. While many studies do not specifically justify their dose selection, a number do cite Reagan-Shaw et al's paper to claim that a sufficient dosage was used to elicit a physiologic response in humans. For example, one review article states, "By using the Reagan-Shaw formula to know the equivalence between these doses and those used in human studies, it can be deduced that the doses used in human studies are in the same range as those used in animal studies (equivalent to 30-2.220 mg/day). Consequently, the reduced response to 
resveratrol in humans cannot be attributed to the use of lower doses in humans than in animals [27]". Therefore, it is clear that conclusions about interspecies differences in physiologic response, and thereby clinical potential, are focused on the notion that Reagan-Shaw's recommendation for "allometric dose translation" are correct. However, a thorough investigation of Reagan-Shaw's recommendations reveals this is not the case.

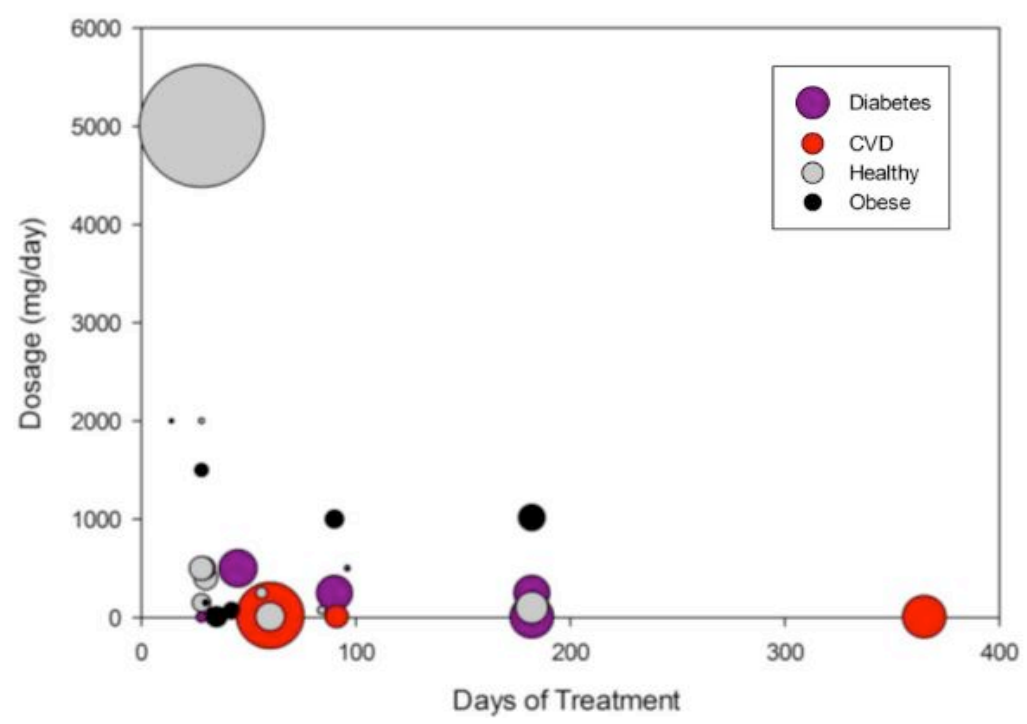

Figure 1.Resveratrol treatment duration, dosage, and sample size of human clinical trials investigating resveratrol. Dot size indicates relative sample size. Most studies use resveratrol dosages $\leq 500 \mathrm{mg} /$ day $(<7 \mathrm{mg} / \mathrm{kg}$ for a $70 \mathrm{~kg}$ person).

\section{MISUNDERSTANDINGS IN ANIMAL-TO-HUMAN DOSE TRANSLATION OF RESVERATROL}

Although the 2008 paper by Reagan-Shaw has been cited $>2,000$ times and is used as a reference for interspecies dose-translation, it is unfortunately rooted in misinterpretation of the scientific literature and its recommendations are unjustified [28]. Reagan-Shaw's basis for their $1.82 \mathrm{mg} / \mathrm{kg}$ calculation is based on their post hoc prediction that scaling an oral dosage by BSA accurately providing an animal-to-human dosage conversion. However, this argument did not consider any of the available pharmacokinetic data necessary for allometric scaling, let alone consider a desired target or physiologic effect. Our review of the modern literature found that it is not acceptable to estimate therapeutic range of a small molecule in humans simply by utilizing the $\mathrm{mg} / \mathrm{kg}$ oral dosage in a single study in mice [3, 26, 29].

Additionally, recommendations for resveratrol dosage have frequently been based on the dosage utilized by Baur et al's 2006 work in health and survivability of mice on a high calorie diet (and scaling of that dosage), which was specific for metabolic applications but not necessarily for other applications (e.g., cardiovascular or neurologic studies) [23]. It is well established that the dosage of a drug may vary, depending on the desired pharmacological effect. For instance, anti-thrombotic dosages of aspirin are different than that required for pain relief, much like the dosages of sildenafil differ between erectile dysfunction and 
pulmonary arterial hypertension patients [30]. Given that a number of potential health benefits have been described for resveratrol, it is not appropriate to suggest one specific dose as a "human equivalent." As a result, it is necessary to consider the pharmacological parameter of interest before recommending a dosage. Measures of rapid $C_{\max }$ within may be important for acute effects, such as applications for ischemic conditions [31]. Maintenance of plasma resveratrol exposure, measured using area under the curve (AUC), is of interest in other effects, including anti-diabetic applications [32]. Because Baur et al did not report pharmacokinetic data, it was not possible to appropriately use allometric scaling for dose determination.

\section{ATTEMPTING TO DEVELOPING A SIMPLE ALLOMETRIC SCALING MODEL FOR RESVERATROL}

In developing their recommendation for developing a human dose, Reagan-Shaw et al failed to present any correlation to the published in vivo pharmacokinetic parameters of resveratrol from scaling by BSA [22]. As a result, predicted dose-responses from allometric could not be compared to observed dose-responses from clinical trials. In the case of resveratrol, the human and rodent pharmacokinetic data had already been published and reviewed by multiple groups before the 2008 paper [2, 33-37]. However, in the decade that has passed since Baur et al's groundbreaking study, the available published data on resveratrol pharmacokinetics remain surprisingly insufficient for developing an allometric model.

An allometric model should theoretically include a response variable (e.g., $C_{\max }$ ) that was obtained across similar conditions across species of varying body mass. In this case, "similar conditions" should be the same dosage (e.g., in $\mathrm{mg} / \mathrm{kg}$ ) and the same delivery methods (e.g., oral administration). Unfortunately, few studies have provided similar dosages across species, and papers from different research groups seldom align in their dosing protocols. Lack of data from consistent dosages between studies can theoretically be overcome by extrapolating a $C_{\max }$ for a given dosage if a dose-curve is reported. Once again, over two decades of laboratory animal research on resveratrol has produced surprisingly very few dose curves of free trans-resveratrol. Moreover, many published studies report their data graphically, rather than in tabular format, which limits its utility for extrapolation.

In an attempt to determine the validity of recommendations for interspecies dosage translation of resveratrol, we attempted to develop an allometric model using existing published data. We were able to obtain $C_{\max }$ data for a $100 \mathrm{mg} / \mathrm{kg}$ oral dosage using data examining resveratrol supplementation in 24 mice from J.J. Johnson et al [38], and data from 8 dogs reported by W.D. Johnson et al [39]. An allometric model based on data from only two species may be considered inadequate; however, sufficient data from other species were not available. Additionally, weaknesses within these datasets could potentially impair the accuracy of the allometric model. For instance, data from J.J. Johnson et al [38] had relatively high standard errors $\left(\sim 33 \%\right.$ of mean) for $C_{\max }$, and variance measures were not reported for AUC. Consequently, it could be argued that this high variability could yield a sub-optimal model. 
Additionally, we had to extrapolate the $C_{\max }$ for a $100 \mathrm{mg} / \mathrm{kg}$ oral dosage in dogs from the dosage range reported $(200-1200 \mathrm{mg} / \mathrm{kg})$. Given the linear relationship between dosage and $C_{\max }$ in dogs (Figure 2), we felt that such extrapolation was reasonably appropriate. However, our extrapolated $C_{\max }$ for a $100 \mathrm{mg} / \mathrm{kg}$ is not within the original range of dosages, which could raise concerns regarding its validity. While the body mass of the dogs was estimated to be $\sim 7.7 \mathrm{~kg}$ from a figure within the text, the body mass (and sex distribution) of mice were not reported, so they were estimated to be $25 \mathrm{~g}$, based on available information elsewhere. Clearly, there are multiple limitations of the existing research. Accordingly, the weakness in our allometric model is already apparent. Nonetheless, this further demonstrates our point that developing a highquality allometric model for selecting a human dosage for resveratrol is not yet possible.

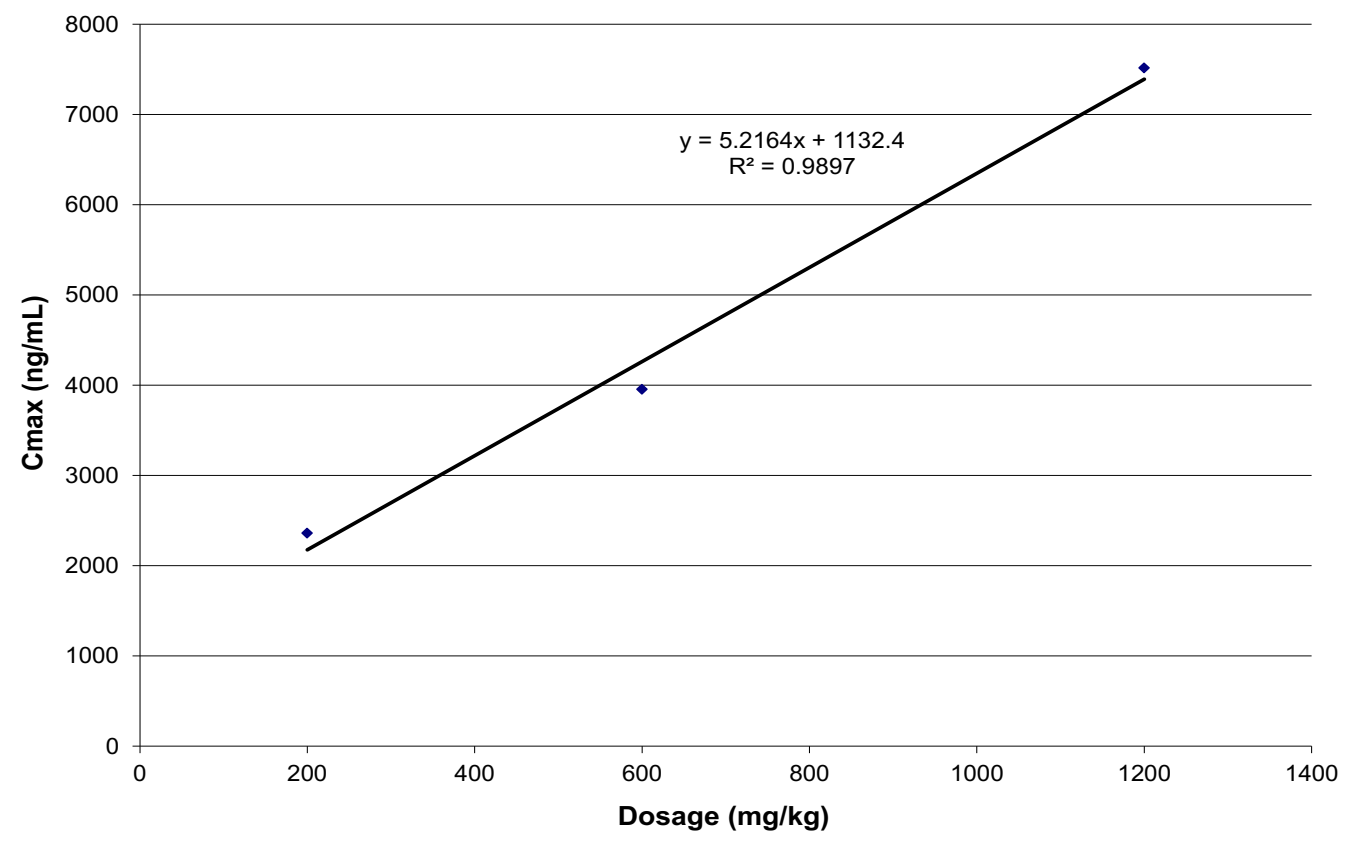

Figure 2.Maximal plasma concentration $\left(C_{\max }\right)$ of free trans-resveratrol in dogs provided three different oral dosages $(200 \mathrm{mg} / \mathrm{kg}, 600 \mathrm{mg} / \mathrm{kg}$, and $1200 \mathrm{mg} / \mathrm{kg})$ of resveratrol, using W.D. Johnson's [39] data. There was a linear relationship across three dosages provided, allowing for the $C_{\max }$ at other dosages to be estimated.

The intercept of our model is $2279.0 \mathrm{ng} / \mathrm{mL}$ and the slope is $-0.0812(\mathrm{ng} / \mathrm{mL}) / \mathrm{kg}$ (Figure 3). For a $70 \mathrm{~kg}$ human, this model predicts a $C_{\max }$ of $921 \mathrm{ng} / \mathrm{mL}$ following a $100 \mathrm{mg} / \mathrm{kg}$ dosage. However, given the aforementioned limitations of the model, there is expected to be considerable error in this model. When extrapolating data from Boocock [2], a $100 \mathrm{mg} / \mathrm{kg}$ dosage for a human would be expected to yield a $C_{\max }$ of $760.9 \mathrm{ng} / \mathrm{mL}$. Thus, the very rough allometric model developed here has about $21 \%$ error, which is actually within the acceptable range for allometry [5]. However, it remains important to emphasize that this model is only that for one pharmacokinetic parameter, $C_{\max }$, and does not necessarily translate to other physiologic parameters, let alone clinical response. 


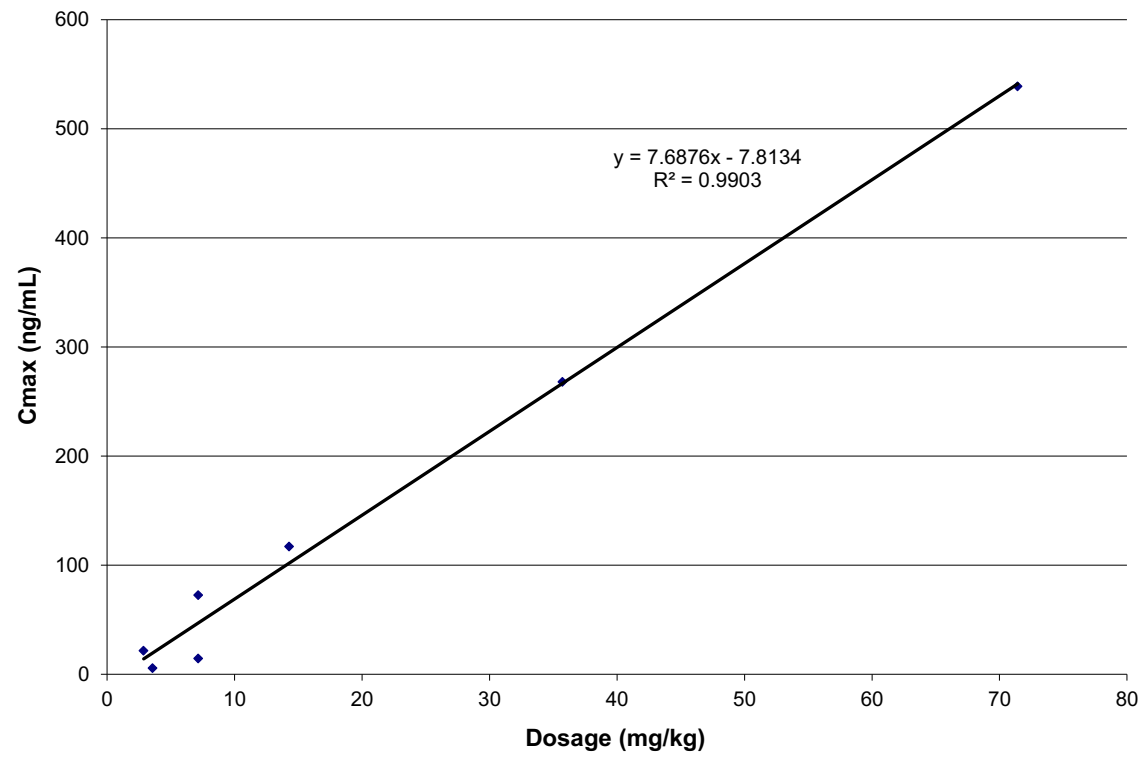

Figure 3. $C_{\max }$ of free trans-resveratrol in humans across multiple dosages, using data from Almeida [1] and Boocock [2]. There was a linear relationship across three dosages provided.

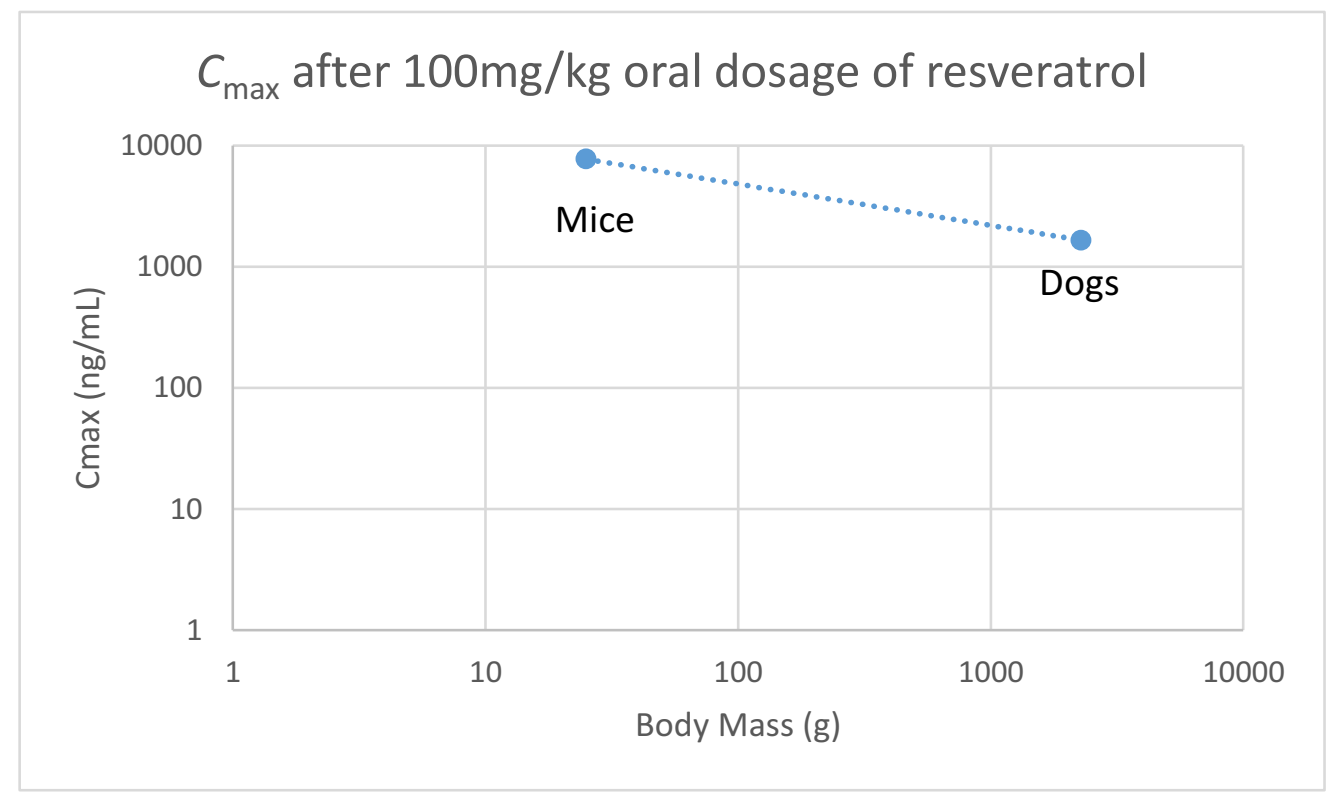

Figure 4. Attempted allometric model to predict $C_{\max }$ of resveratrol following $100 \mathrm{mg} / \mathrm{kg}$ oral dosage, using data from J.J. Johnson in mice, and W.D. Johnson in dogs

While our rough allometric model (based on extrapolations from only two species) seems reasonable in predicting $C_{\max }$ across species, it is dangerous to not take interspecies differences into consideration $[25,26]$. This is nicely demonstrated when comparing existing dose-response data between humans and dogs. The relationship between oral dosage of resveratrol and $C_{\max }$ is strongly linear in both dogs $\left(R^{2}=0.9897\right.$, Figure 2$)$ and humans $\left(R^{2}=0.9903\right.$, Figure 4$)$. This linear relationship allows for the $C_{\max }$ for a given dosage to be estimated in each species. Using 
data from Johnson [39], a $32 \mathrm{mg} / \mathrm{kg}$ dosage of resveratrol should yield a $C_{\max }$ of $\sim 1300 \mathrm{ng} / \mathrm{mL}$ in dogs. Based on the allometric dose translation suggested by Reagan-Shaw, the "equivalent" of a $32 \mathrm{mg} / \mathrm{kg}$ dosage in dogs should be approximately $17.3 \mathrm{mg} / \mathrm{kg}$ in humans (or $\sim 1200 \mathrm{mg}$ in a $70 \mathrm{~kg}$ human). However, data from Boocock et al [2] reveals that providing humans with a similar (1000mg) dosage results in a $C_{\max }$ of $117 \mathrm{ng} / \mathrm{mL}$, or about $10 \%$ of what would be predicted if it were truly an "equivalent" dose. Even when humans are provided 5000mg of resveratrol (approximately $71 \mathrm{mg} / \mathrm{kg}$ for a $70 \mathrm{~kg}$ individual), the $C_{\max }$ is $538.8 \mathrm{ng} / \mathrm{mL}$. In other words, providing humans with more than double the dosage results in less than half of the plasma concentration achieved in dogs. This clearly demonstrates that there are interspecies differences in resveratrol pharmacokinetics, and that the simple BSA formula presented by Reagan-Shaw et al does not accurately predict pharmacokinetic parameters. Although the $22 \mathrm{mg} / \mathrm{kg}$ dose recommended by Baur was called "unfeasibly high" by proponents of Reagan-Shaw's recommendation [40], the literature demonstrates that this dosage is realistic and safe [41, 42]. Furthermore, in the case of diabetes, higher dosages at or above this range have been demonstrated to be beneficial in human patients [43].

From the resveratrol example alone, it is clear that applying a BSA-based "allometric dose translation" to convert between an animal dosage and a human dosage does not yield similar pharmacokinetic responses. This is further exemplified through the extrapolation methods we used for estimating the pharmacokinetic response to a $100 \mathrm{mg} / \mathrm{kg}$ dosage in dogs. Although there are strong linear relationships $\left(\mathrm{R}^{2}>0.95\right)$ between dosage and $C_{\max }$, and dosage and AUC in dogs, extrapolating to a $100 \mathrm{mg} / \mathrm{kg}$ dosage yields a physiologically impossible negative value for the AUC. From this, and other examples presented here, it is clear that there is insufficient data available to properly develop an allometric dose translation. A major reason for this is that papers from animal model experiments have not reported pharmacokinetic parameters or physiologic responses to consistent dosages across a variety of species, or dose-response curves. Additionally, there may be considerable inter-individual variability in pharmacokinetic parameters in response to a given dosage, which creates further barriers for accurate model development.

Although there has been considerable confusion regarding the "allometric dose translation" of resveratrol, it may now be considered a moot point. Since there is now pharmacokinetic data from humans available, beginning with Boocock's 2007 publication, estimates from animal models are no longer necessary for general evaluation of resveratrol interventions. However, it is possible that specific physiologic responses to resveratrol which cannot be easily or noninvasively measured in humans may still benefit from proper allometric scaling.

\section{LIMITATIONS OF BSA SCALING IN INTERSPECIES DOSAGE TRANSLATION}

While the previous discussion uses data from animal models and humans to demonstrate that a dosage of resveratrol cannot simply be scaled by using BSA, it does not address some of the further misunderstandings and misinterpretation regarding BSA scaling and its applications. While BSA has been used extensively as an animal-to-human scaling factor for first-in-human dosages from a bureaucratic tradition, its use across multiple applications is not fully justified. Reagan-Shaw et al state that "...the Food and Drug Administration has suggested that the extrapolation of animal dose to human dose is correctly performed only through normalization 
to BSA..." This erroneously states that the US Food and Drug Administration provides guidelines stating that BSA properly converts dosages from animal trials which will show therapeutic effect in human trials. In the glossary of the FDA guidelines cited, the FDA clearly states a difference between their definitions for Human Equivalent Dose (HED) and Pharmacologically Active Dosage (PAD)[44].

According to the FDA guidelines, the definition of HED is based on the no observable adverse event level (NOAEL) or the no observable event level (NOEL)[44]. Conversion of a drug dosage from model species to humans by the HED by the FDA is not inherently expected to not elicit a physiological effect or pharmacologically therapeutic dosage [44]. This is clarified in the updated version of the FDA guidelines for safe starting dosages in humans from 2005[45]. In other words, it should be clear that "human equivalent dose" is too vague to establish PAD, as it does not consider pharmacokinetic parameters, such as $C_{\max }$ or AUC, which are key factors for eliciting an intervention's effect on its cellular target [22, 29]. Plasma level and drug exposure are measures closely related to tissue distribution, which is desired to allow contact between drug and desired target of action. These drugs to the targets of action follow the law of mass action of binding, where the concentration of drug needs to be in the desired tissue at the amount to allow binding to the desired target. Therefore, according to FDA guidelines, it is not appropriate to use BSA scaling to convert the $22 \mathrm{mg} / \mathrm{kg}$ dosage used by Baur et al (which produced a physiologic response) into a human PAD.

In the discussion of BSA by Reagan Shaw et al, it would appear that these works advocate the continued use of BSA in clinical practice [46-49]. A brief review of these works reveals the opposite. For example, Sawyer and Ratain report a poor correlation between body surface area and chemotherapy doses in children, with the drug more closely relating to body weight than to BSA in many cases [47]. They appropriately examine scaling for a pharmacokinetic variable of interest (renal clearance), not just a general dosage. Regardless, that reference is about cytotoxic drug dosing and scaling between different size individuals in the same species, rather than interspecies scaling. The conclusions of Sawyer and Ratain state: "BSA was introduced in medical oncology to safely predict a suitable starting dose in phase I clinical trials from preclinical animal toxicology data. From that starting point in phase I trials it has spread throughout the practice of oncology with little justification.

Similar concerns about BSA scaling are raised by other references cited by Reagan-Shaw et al. For instance, Baker et al. discusses cytotoxic drug dose selection for phase I clinical trials, but does not suggest dose selection for therapeutic effect. Reagan-Shaw implies that Baker et al. advocate for the use of "an average $1.86 \mathrm{~m}^{2}$ BSA" for calculating a pharmacologically active dose between species [22, 49]. Baker et aldoes not actually discuss an exploration of the therapeutic range of cytotoxic drugs, but simply for the phase I clinical trial for estimating drug toxicity. As for the therapeutic effect, Baker et al states, "Although the primary objective of phase I trials is to evaluate drug toxicity, antitumor activity is usually a secondary objective. Other measures, such as drug clearance, have also been used as surrogate markers of drug effects." Baker also discusses further issues with BSA, in which they ultimately state the scientific rational for its use is lacking [49]. The overall theme of Baker is the discussion of the rationale of BSA in selecting the appropriate dosage between human patients, in addition to not considering interspecies pharmacokinetics or scaling [49]. Similar criticisms and concerns are 
discussed within other references cited, including Kaestner and Sewell[46] as well as Gurney [48].

\section{SUMMARY AND CONCLUSIONS}

Allometric scaling, when used properly, is a well-established method of estimating pharmacokinetic parameters and physiologic responses across species. The accuracy of the estimates varies considerably for different drugs of interest, but corrective factors enhance its applicability. While more accurate estimation methods are available, allometric scaling is generally inexpensive and easy to perform for pilot trials. Although allometric scaling utilizing BSA in dose translation has been suggested for converting the pharmacologically active dosage of resveratrol from rodent to human studies, the chief source of this recommendation is inherently flawed for a number of reasons. When designing human clinical trials based on in vitro to in vivo studies, it is imperative to consider pharmacokinetic data and/or physiologic responses. This is verified through examining the predicted and actual values of pharmacokinetic data measured in human resveratrol interventions. The resveratrol example should serve a cautionary tale for improperly using allometry or BSA scaling when determining the dosages to achieve desired physiological effects for other xenobiotics and nutraceuticals. Likewise, while body surface area is recommended for establishing a safe starting dose for firstin-human studies, rather than a pharmacologically active dose, a science-based justification for this method's utility over other scaling procedures is dubious. Although there have been recommendations for a human dosage of resveratrol based on supposed allometric scaling practices, a true allometric model of the pharmacokinetics of resveratrol is actually quite difficult to develop for humans because of surprisingly inadequate data reported in the literature from animal models. This is also likely the case for other natural products. However, the availability of actual data from human clinical trials negates the need to perform interspecies allometric scaling for most clinical trials involving resveratrol.

\section{Abbreviations}

PBPK, physiologically-based pharmacokinetic modeling; BSA, body surface area; FDA, United States Food and Drug Administration; AUC, area under the curve; $C_{\max }$, maximum plasma concentration; HED, human equivalent dose; PAD, pharmacologically active dose; NOAEL, no observable adverse events limit

\section{Competing interests}

Otis L. Blanchard is the Chief Technology Officer of Ace Laboratories Inc, Seattle, WA \& Owner of Wilmore Laboratories, LLC. Ace Laboratories and Wilmore Laboratories have financial interest in a patent $(9,125,858)$ and patent application $(14 / 691,320)$ regarding novel resveratrol pharmaceutical formulations. James M. Smoliga is Chair of the Scientific Advisory Board for Ace Laboratories, Inc.

\section{Authors' contributions}

James M. Smoliga and Otis L. Blanchard contributed equally to this manuscript. Both authors drafted individual sections of this manuscript, and both authors contributed to revising all 
contents. JMS had primary responsibility for the final content. Both authors have read and approved the final manuscript.

\section{Acknowledgements and funding}

The authors have not received any funding to support this work.

\section{REFERENCES:}

1. Almeida L, Vaz-da-Silva M, Falcao A, Soares E, Costa R, Loureiro AI, et al. Pharmacokinetic and safety profile of trans-resveratrol in a rising multiple-dose study in healthy volunteers. Molecular nutrition \& food research. 2009;53 Suppl 1:S7-15.

2. Boocock DJ, Faust GES, Patel KR, Schinas AM, Brown VA, Ducharme MP, et al. Phase I dose escalation pharmacokinetic study in healthy volunteers of resveratrol, a potential cancer chemoprotective agent. Cancer Epidemiology, Biomarkers \& Prevention. 2007;16(6):1246-52.

3. Fan J, de Lannoy IA. Pharmacokinetics. Biochemical pharmacology. 2014;87(1):93-120.

4. Jones HM, Dickins M, Youdim K, Gosset JR, Attkins NJ, Hay TL, et al. Application of PBPK modelling in drug discovery and development at Pfizer. Xenobiotica. 2012;42(1):94-106. doi: 10.3109/00498254.2011.627477. PubMed PMID: 22035569.

5. Mahmood I. Interspecies pharmacokinetic scaling. Rockville, MD: Pine House Publishers; 2005.

6. Von Bertalanffy L. Quantitative laws in metabolism and growth. The Quarterly review of biology. 1957;32(3):217-31.

7. Lin JH. Applications and limitations of interspecies scaling and in vitro extrapolation in pharmacokinetics. Drug metabolism and disposition: the biological fate of chemicals. 1998;26(12):1202-12.

8. Mahmood I, Balian JD. The pharmacokinetic principles behind scaling from preclinical results to phase I protocols. Clinical pharmacokinetics. 1999;36(1):1-11

9. Kleiber M. Body size and metabolic rate. Physiological reviews. 1947;27(4):511-41.

10. 10Mahmood I. Application of allometric principles for the prediction of pharmacokinetics in human and veterinary drug development. Advanced drug delivery reviews. 2007;59(11):1177-92

11. Mahmood I, Balian JD. Interspecies scaling: predicting clearance of drugs in humans. Three different approaches. Xenobiotica; the fate of foreign compounds in biological systems. 1996;26(9):887-95.

12. Tang H, Mayersohn M. A mathematical description of the functionality of correction factors used in allometry for predicting human drug clearance. Drug metabolism and disposition: the biological fate of chemicals. 2005;33(9):1294-6

13. Mahmood I. Comments on "a mathematical description of the functionality of correction factors used in allometry for predicting human drug clearance". Drug metabolism and disposition: the biological fate of chemicals. 2006;34(3):507-9; author reply 10-1.

14. Zou P, Zheng N, Yu Y, Yu S, Sun W, McEachem D, et al. Preclinical pharmacokinetics of MI-219, a novel human double minute 2 (HDM2) inhibitor and prediction of human 
pharmacokinetics. Journal of pharmacy \& pharmaceutical sciences : a publication of the Canadian Society for Pharmaceutical Sciences, Societe canadienne des sciences pharmaceutiques. 2012;15(2):265-80.

15. Takaoka M. The phenolic substances of white hellebore (Veratrum Grandiflorum Loes fil.) II. Nippon Kagaku Kaishi. 1939;60(12):1261-4.

16. Langcake P, Pryce R. The production of resveratrol by Vitis vinifera and other members of the Vitaceae as a response to infection or injury. Physiological Plant Pathology. 1976;9(1):77-86.

17. Siemann EH, Creasy LL. Concentration of the Phytoalexin Resveratrol in Wine. American Journal of Enology and Viticulture. 1992;43:49-52.

18. Jang M, Cai L, Udeani GO, Slowing KV, Thomas CF, Beecher CW, et al. Cancer chemopreventive activity of resveratrol, a natural product derived from grapes. Science (New York, NY). 1997;275(5297):218-20.

19. Howitz KT, Bitterman KJ, Cohen HY, Lamming DW, Lavu S, Wood JG, et al. Small molecule activators of sirtuins extend Saccharomyces cerevisiae lifespan. Nature. 2003;425(6954):191-6.

20. Wood JG, Rogina B, Lavu S, Howitz K, Helfand SL, Tatar M, et al. Sirtuin activators mimic caloric restriction and delay ageing in metazoans. Nature. 2004;430(7000):686-9.

21. Baur JA, Pearson KJ, Price NL, Jamieson HA, Lerin C, Kalra A, et al. Resveratrol improves health and survival of mice on a high-calorie diet. Nature. 2006;444(7117):33742.

22. Reagan-Shaw S, Nihal M, Ahmad N. Dose translation from animal to human studies revisited. FASEB journal : official publication of the Federation of American Societies for Experimental Biology. 2008;22(3):659-61

23. Baur JA, Pearson KJ, Price NL, Jamieson HA, Lerin C, Kalra A, et al. Resveratrol improves health and survival of mice on a high-calorie diet. Nature. 2006;444(16):33742.

24. Baur JA, Sinclair DA. Therapeutic potential of resveratrol: the in vivo evidence. Nat Rev Drug Discov. 2006;5(6):493-506.

25. de Jong M, Maina T. Of mice and humans: are they the same?--Implications in cancer translational research. Journal of nuclear medicine : official publication, Society of Nuclear Medicine. 2010;51(4):501-4.

26. Mak IW, Evaniew N, Ghert M. Lost in translation: animal models and clinical trials in cancer treatment. American journal of translational research. 2014;6(2):114-8.

27. Fernandez-Quintela A, Carpene C, Fernandez M, Aguirre L, Milton-Laskibar I, Contreras $\mathrm{J}$, et al. Anti-obesity effects of resveratrol: comparison between animal models and humans. Journal of physiology and biochemistry. 2016.

28. Blanchard OL, Smoliga JM. Translating dosages from animal models to human clinical trials--revisiting body surface area scaling. FASEB journal : official publication of the Federation of American Societies for Experimental Biology. 2015;29(5):1629-34.

29. Muller PY, Milton MN. The determination and interpretation of the therapeutic index in drug development. Nat Rev Drug Discov. 2012;11(10):751-61 
30. Wirostko BM, Tressler C, Hwang LJ, Burgess G, Laties AM. Ocular safety of sildenafil citrate when administered chronically for pulmonary arterial hypertension: results from phase III, randomised, double masked, placebo controlled trial and open label extension. BMJ. 2012;344:e554.

31. Gordish KL, Beierwaltes WH. Resveratrol induces acute endothelium-dependent renal vasodilation mediated through nitric oxide and reactive oxygen species scavenging. American journal of physiology Renal physiology. 2014;306(5):F542-50. Epub 2014/01/17

32. Elliott P, Walpole S, Morelli L, Lambert P, Lunsmann W, Westphal C, et al. Resveratrol / SRT-501. Drugs of the Future. 2009;34(4):291-5.

33. Walle T, Hsieh F, DeLegge MH, John E Oatis J, Walle UK. High absorption but very low bioavailability of oral resveratrol in humans. Drug Metabolism and Disposition. 2004;32(12):1377-82.

34. Asensi M, Medina I, Ortega A, Carretero J, Bano MC, Obrador E, et al. Inhibition Of Cancer Growth By Resveratrol Is Related To Its Low Bioavailability. Free Radical Biology \& Medicine. 2002;33(3):387-98.

35. Marier JF, Vachon P, Gritsas A, Zhang J, Moreau JP, Ducharme MP. Metabolism and disposition of resveratrol in rats: extent of absorption, glucuronidation, and enterohepatic recirculation evidenced by a linked-rat model. The Journal of pharmacology and experimental therapeutics. 2002;302(1):369-73.

36. Gescher AJ, Steward WP. Relationship between mechanisms, bioavailibility, and preclinical chemopreventive efficacy of resveratrol: a conundrum. Cancer epidemiology, biomarkers \& prevention : a publication of the American Association for Cancer Research, cosponsored by the American Society of Preventive Oncology. 2003;12(10):953-7.

37. Goldberg DM, Yan J, Soleas GJ. Absorption of three wine-related polyphenols in three different matrices by healthy subjects. Clinical Biochemistry. 2003;36(1):79-87.

38. Johnson JJ, Nihal M, Siddiqui IA, Scarlett CO, Bailey HH, Mukhtar H, et al. Enhancing the bioavailability of resveratrol by combining it with piperine. Molecular nutrition \& food research. 2011;55(8):1169-76.

39. Johnson WD, Morrissey RL, Usborne AL, Kapetanovic I, Crowell JA, Muzzio M, et al. Subchronic oral toxicity and cardiovascular safety pharmacology studies of resveratrol, a naturally occurring polyphenol with cancer preventive activity. Food and chemical toxicology : an international journal published for the British Industrial Biological Research Association. 2011;49(12):3319-27.

40. Sharma V, McNeill JH. To scale or not to scale: the principles of dose extrapolation. Br J Pharmacol. 2009;157(6):907-21.

41. Boocock DJ, Patel KR, Faust GE, Normolle DP, Marczylo TH, Crowell JA, et al. Quantitation of trans-resveratrol and detection of its metabolites in human plasma and urine by high performance liquid chromatography. J Chromatogr B Analyt Technol Biomed Life Sci. 2007;848(2):182-7.

42. Brown VA, Patel KR, Viskaduraki M, Crowell JA, Perloff M, Booth TD, et al. Repeat Dose Study of the Cancer Chemopreventive Agent Resveratrol in Healthy Volunteers: 
Safety, Pharmacokinetics, and Effect on the Insulin-like Growth Factor Axis. Cancer Res. 2010;70(22):9003-11.

43. Hausenblas HA, Schoulda JA, Smoliga JM. Resveratrol treatment as an adjunct to pharmacological management in type 2 diabetes mellitus--systematic review and metaanalysis. Mol Nutr Food Res. 2015;59(1):147-59.

44. FDA. Draft Guidance for Industry and Reviewers on Estimating the Safe Starting Dose in Clinical Trials for Therapeutics in Adult Healthy Volunteers. 2002.

45. FDA. Guidance for Industry: Estimating the Maximum Safe Starting Dose in Initial Clinical Trials for Therapeutics in Adult Healthy Volunteers. 2005.

46. Kaestner SA, Sewell GJ. Chemotherapy dosing part I: scientific basis for current practice and use of body surface area. Clinical oncology. 2007;19(1):23-37.

47. Sawyer M, Ratain MJ. Body surface area as a determinant of pharmacokinetics and drug dosing. Investigational new drugs. 2001;19(2):171-7.48. Gurney H. How to calculate the dose of chemotherapy. British journal of cancer. 2002;86(8):1297-302.

48. Baker SD, Verweij J, Rowinsky EK, Donehower RC, Schellens JH, Grochow LB, et al. Role of body surface area in dosing of investigational anticancer agents in adults, 19912001. Journal of the National Cancer Institute. 2002;94(24):1883-8. 